Frage der variskischen Tektonik lösen sie aber auch noch nicht; sie haben nur das Verdienst, daß sie uns zwingen, auch das übrige Materia? einer Prüfung zu unterziehen. Einen weiteren $Z$ weck verfolgt auch diese Mitteilung über die Münchberger Gneismasse nicht.

Alle diese Probleme, die uns das Elbtalgebirge oder die Randfaltungen der Böhmischen Masse in NW und SO (morawisch-moldanubisch) zeigen, sind ebenso wie Fragen nach der Entstehung der Leberauer Grauwackè und des Talhorns in den Vogesen, oder der gepreßten Granite von Alt-Glashütten im Schwarzwald alles Teile eines komplexen Erscheinungsbildes. Unser Streben, diese Erscheinungen zu verstehen und zu erklären, bleibt einstweilen nur ein tastender Versuch, den Schleier des Geheimnisses zu lïften, der den Mechanismus der Gebirgsfaltung zwischen Oberdevon und Oberkarbon in unseren deutschen Gebirgen noch verdeckt.

\title{
Ein Beitrag zur Gebirgsbildung des Varistischen Bogens.
}

\section{Von A. Born-Frankfurt a. M.1)}

Die Frage nach dem Mechanismus der Gebirgsbildung im weitesten Sinne findet ihre Antwort in der Erkenntnis des Aufbaues der Gebirge. Die jüngeren Faltengebirge von alpinem Alter haben bisher zur Lösung dieser Frage wenig beigetragen. Die Ursachen hierfür sind vor allem in ihrem Aufbau begründet. Der Deckenbau des alpinen Gebirgstyps ist ein zu komplizierter, als daß aus ihm leicht die Antwort auf die Frage nach der Art des Verlaufs hätte entnommen werden können. Generationen von Forschern haben ihre Kraft eingesetzt, um allein zu einer klaren Erkenntnis der Lagerungsformen zu gelangen. Erst jetzt beginnt die Ernte zu reifen (E. ARGAND, R. STAUB). Ein weiterer Grund des Versagens liegt in der Jugendlichkeit der Gebirge, in dem geringen Maß der Abtragung und Enthïllung des Tiefenmechanismus. Einfacher gebaute Faltengebirge jüngeren Datums, wie sie sich außereuropäisch verbreitet finden, sind theoretischen Erwägungen über den Mechanismus ihrer Entstehung nicht unzugänglich. W. PENcK gelang es neuerdings in den argentinischen Anden aus dem Verhältnis von Gebirgsbau zu Vulkanismus Vorstellungen über den Werdegang der Gebirgsbildung und seine Ursachen zu entnehmen (Abh. sächs. Ges. Wiss., math.-phys. K1., Bd. 37, 1. 1920).

Bildungen älterer orogenetischer Phasen sind entweder nur fragmentarisch erhalten bzw. zugänglich, oder in den Einzelheiten des Aufbaues und der Sédimentation zu wenig gellärt, um für theoretische

1) Erweiterte Form eines im März 1920 auf der Hauptversammlung der Geologischen Vereinigung in Gießen gehaltenen Vortrags. 
Erörterungen reif zu sein. Andrerseits bieten sie einen wesentlichen Vorteil: weitgehende Abtragung hat zur Entblößung tieferer Teile geführt, so daß Einblicke in sonst unzugängliche Tiefen und dadurch Schlüsse auf den Tiefenmechanismus ermöglicht werden.

Wenn im folgenden Utberlegungen zur Gebirgsbildung des Varistischen Bogens angestellt werden, möchte ich damit nicht zum Ausdruck bringen, daß das Stadium der Erforschung des Varistischen Bogens reif für theoretische Erwägungen genannter Art ist. Mebr denn je sind wir uns im klaren, daß die Erkenntnis der Lagerungsformen und noch mehr die der Sedimentationsbedingungen im anfänglichen Stadium einer exakten Erforschung steht. Aber schon die klare Erkenntnis der Probleme allein erleichtert den Weg zu ihrex Lösung. Von diesem Gesichtspunkt aus möchte ich die folgenden Zeilen verstanden wissen.

Für die anschließenden Utberlegungen werde ich mich im wesentlichen auf einen Sektor aus dem Varistischen Bogen beschränken, der gegeben ist durch die oberrheinischen Massive Schwarzwald, Vogesen, Hardt und Odenwald, und durch die Rheinische Masse, ein Sektor, der durchgreift von den inneren Teilen des Bogens bis zum ungefalteten Außenrand, und den ich als rheinischen Sektor des Varistischen Bogens bezeichne.

Wir knüpfen die Bildung der Faltengebirge an die Präexistenz von Geosynklinalen, worunter ich im Sinne von H. STILLE säkular sinkende Sedimentationsräume verstanden wissen möchte. Die Geosynklinale des Varistischen Bogens umfaßt gemäß der herrschenden Auffassung Rheinische Masse, Harz, Ostthüringen, und setzt sich über Sachsen in schlesisches Gebiet fort. In dieser Zone herrschte zur Zeit des Unterkarbon, Devon und zumeist des Silur Meeresbedeckung und rege Sedimentation. Die Mächtigkeitsbeträge gehen in die Tausende, die fazielle Ausbildung ist meist landnahe, so daß ein epirogenetisches Sinken des Meeresbodens selbstverständliche Voraussetzung ist. Gelegentlich wurde die Bewegungsrichtung unterbrochen durch solche aufsteigender Art. Das Devon des Rheinischen Schiefergebirges gestattet aus seinen Sedimenten das Auftreten von episodischen Hochgebieten abzulesen. R. WHDEKIND schloB jüngst aus der faziellen Ausbildung des höheren Mitteldevons, besonders aus der saumriffartigen Anordnung der Korallenkalke, und ebenso aus der sandigen Ausbildung gewisser Oberdevonhorizonte (Pönsandstein), auf die Existenz eines Hochgebietes im Siegerlande, des sog. Siegerländer Blocks (Nachr. Ges. Wiss. Götting., math.-phys. Kl. 1919). Und J. AHLBuRGs Untersuchungen ergaben im Dill-Lahn-Gebiet für die beide Mulden umrandenden Gebiete eine Anlage bereits in mitteldevonischer Zeit (Jahrb. preuß. geol. Landesanst. Bd. 40. 1919, 1). Nach diesen und anderen Beobachtungen ergibt sich hier eine Vorstellung, wie sie jüngst in den Alpen von E. ARGAND und R. Staub gewonnen wurde: Abhängigkeit der heute vorliegenden tektonischen Einheiten von der Sedimentation. In den Alpen wurde 
die embryonale Anlage der Decken als getrennte Sedimentationsräume in frühmesozoische Zeit zurückdatiert. Im Rheinischen Schiefergebirge sind tektonische Hochgebiete (Siegerland, Hörre u. a.) mindestens seit mitteldevonischer Zeit als solche morphologischer Art tätig gewesen. Die Tektonik erweist sich immer mehr als eine Funktion der paläogeographischen Verhältnisse zur Zeit der Sedimentation. Das gilt nicht nur von den beiden Extremen, im großen von der Geosynklinale, und im kleinsten von den Elementen der Falten, sondern auch von den größeren tektonischen Einheiten, Komplexen, die ein Faltengebirge aufbauen, wie die genannten: Lahnmulde, Dillmulde, Hörrezug, u. a. Solche Differenzierung in früher Zeit der Sedimentation widerspricht nicht dem Charakter der Geosynklinale.

Als Hochgebiet, das im Norden Grenze und Detrituslieferant der varistischen Geosynklinale war, betrachten wir das nordatlantische Festland." Wie aber lagen die Verhältnisse im Süden? Offenbart sich in den Oberrheinischen Massiven überhaupt echtes Faltengebirgsland, das aus geosynklinalen Sedimentationsräumen aufstieg?

Die Rolle, die Süddeutschland während der Zeit des Silurs, Devons und Karbons spielte, wird uns zunächst beschäftigen. Den Ausgangspunkt mag die böhmische Masse bilden, deren zentraler Teil als $A b s c h l u B$ einer nicht gerade landnahen altpaläozoischen Sedimentserie im höheren Mitteldevon die mehrere hundert Meter mächtige Schichtenfolge von sandigen Schiefern von Hostim (H 1) mit quarzitischen Einlagerungen (H 2) zeigt. Fragt man nach der Terkunft der sandigen Komponente und der Pflanzeneinschwemmungen im böhmischen Mitteldevon, so liegt die Antwort in der Umrandung der böhmischen Masse.

Im NW und $\mathrm{N}$ treffen wir in Ostthüringen-Fichtelgebirge marines Mitteldevon als Knollenkalk, Tentaculiten- und Nereitenschichten (vgl. u. a. K. Wastrere, N. J. f. Min. Beil.-Bd. 24. 1907. 221); in Sachsen bietet sich das gleiche Bild (F. Kossmat, Geologie von Sachsen. 1916). Im Osten kennen wir Stringocephalenkalk von Olmütz. In allen Gebieten dauert die marine Sedimentation bis in das Oberdevon an. So bleibt als Lieferant des groben terrigenen Detritus Zentralböhmens der. W und SW Süddeutschlands ${ }^{1}$ ).

Was die Oberrheinischen Massive an paläozoischem Sediment überliefern, ist sehr lückenhaft, sowohl horizontal wie vertikal. Auch hier scheint das auf den heutigen Tag Uberlieferte kein zufälliger Erosionsrest zu sein, sondern in manchen Zügen recht gut die Verteilung

1) Keineswegs war jedoch die mittel-oberdevonische Sedimentation in der Umrandung der Böhmischen Masse lückenlos. Hochgebietskerne stecken hier wie andernorts, so z. B. im Bereich der preußischen Oberlansitz, wo K. Prwtzsch (Z. d. D. g. G. 1909, S. 81) den Nachweis erbrachte, daB Kulmkonglomerate mit silurischen Komponenten Silur überlagern. Als Lieferanten der zentralböhmischen Sandmassen dürften solche lokalen Kerne kaum in Betracht kommen. 
der Sedimentations- und Hochgebiete wiederzugeben. Anstehendes Kambrium und Silur fehlen. Eher als der vereinzelte Graptolith, der im alten Diluvium von Villingen als Geröll gefunden wurde (W. DEECKE, Geologie von Baden. Bd. 1. 60-61), lassen die rein obersilurischen Graptolithenformen einen Schluß auf ehemals anstehendes Obersilur za, die E. Nowu (Bull. séanc. soc. d. sc. de Nancy, sér. 3. Bd. 4. 1905. 46-73) als Geröll aus dem Vogesensandstein der westlichen Vogesen beschrieb. Der transgressive Charakter des Obersilurs würde damit auch in den Vogesen zum Ausdruck kommen. Vom Devon fehlt aus dem Schwarzwald und Odenwald jede sichere Utberlieferung. In den Vogesen liegt einwandfreies Mitteldevon im Breuschtal, sowohl tieferes mit Calceola sandalina als höheres mit Stringocephalus Burtini (O. JAEKEL, Mitt. geol. Kommiss. Els.-Lothr. Bd. 1. 1888. 229; W. BüCKING, Mitt. geol. Landesanst. Els.-Lothr. Bd. 12. 1918. 52); beide in landnaher Fazies, erstere als Schiefer und Konglomerate, letztere in Form von Riffkalken und Kalkbreccien als Flachseeabsätze und Schutthalden am Rande der Riffe. Es scheint, daß dem Mitteldevon auch die Tonschiefer (unten) und Kalke nebst Diabasen (oben) zuzurechnen sind, die in den Südvogesen zwischen Chagey und Chennebier, nördlich Héricourt sattelförmig auftreten. Die Kalke fand ich hier nicht als Riff-, sondern als Plattenkalke ausgebildet.

Abgesehen von karbonischen Vorkommen fehlt jedes weitere sichere Zeugnis paläozoischer Meeresbedeckung in Schwarzwald-Vogesen wie Süddeutschland überhaupt. Alle metamorphen Sedimentgesteine, wie die des Odenwaldes, die des Draisbach- und Eberbachtales von BadenBaden, die der Zone Schönau-Badenweiler im südlichen Schwarzwald, ferner die Steiger und Weiler Schiefer der Vogesen und ähnliches sind in ihrer Altersstellung gänzlich ungewib und jeder Spekulation preisgegeben.

Jüngste Zeugen paläozoischer Meeresbedeckung in Süddeutschland sind die Sedimente des Unterkarbons im Südschwarzwald und in den Südvogesen. Aus ersterem sind es schwarze Schiefer von Schönau mit Prolecanites cf. Lyoni (SpIngelmalter, Centralbl. f. Min. 1910), einer Form, die als Prolecanites ältestes Unterkarbon andeuten könnte, spezifisch aber keinerlei stratigraphische Bedeutung hat. Die gesamte Unterkarbonserie ist hier ziemlich mächtig. Wieviel von dem, was S. v. BubNoFr (Oberrh. geol. Ver., N. F. Bd. 8. 1919. 28) zum Unterkarbon stellt, als marin aufzufassen ist, wäre erst durch weitere Fossilfunde zu entscheiden. Auf jeden Fall würde es sich um außerordentlich landnahe Bildungen handeln.

Eine.an Mächtigkeit weit umfangreichere Serie - L. v. WERVEKE schätzt die Gesamtmächtigkeit auf ca. $13000 \mathrm{~m}$ (Führer d. d. Elsaß, 365) - liegt in den südlichen Vogesen. Ergußgesteine nehmen daran wesentlichen Anteil (7000 m). Soweit es sich um Gesteine aquatischer Natur handelt, sind sie meist von grobem terrigenen Detritus. Epiro- 
genetische Senkung ist die notwendige Voraussetzung für diesen Sedimentationsraum. Das einzige rarine Anzeichen, die reiche Fauna von. la Boutique bei Oberburbach (ToRNQUIST, Abh. geol. Spez.-Karte v. Els.-Lothr. Bd. 5. 1895. 97) liegt an der Grenze einer nittleren und oberen Stufe (S. v. BubNofr, 1. c. 31) und hat das Alter des höheren Viséen, genauer der Productus giganieus-Zone. Was wir im Südschwarzwald und in den Südvogesen an Unterkarbon antreffen, macht den Eindruck von am Rande eines Hochgebietes in sinkenden Räumen niedergelegtem Festlandsschutt, in dië gelegentlich bei günstigen Verhältnissen marine Faunen einwanderten.

Was an paläozoischem Sediment postunterkarbonisch zur Ablagerung kam, ist rein kontinentaler Gebirgsschutt oberkarbonen und rotliegenden Alters, angesammelt in den I epressionen der damaligen Hochgebiete. Die Reihe der marinen paläozoischen Sedimente ist also für Süddeutschland sehr lückenhaft. Es darf jedoch nicht vergessen werden, daß metamorphosierende Faktoren vor allem im Karbon in großer Verbreitung auftreten, und daß manche der metamorphen Gesteine ungewissen Alters bisher nicht nachgewiesener Formationsglieder sein oder eine weitere Verbreitung bereits belzannter Schichtenglieder erweisen könnten 1).

Für die Beurteilung Süddeutschlands während paläozoischer Zeit ist ein weiterer Gesichtspunkt von Bedeutung. Soweit es sich für die vorhandenen Vorkommen von Paläozoikum mit Sicherheit feststellen läßt, ruhen sie alle auf weit älterem Gestein und enthalten zwischen sich und der Unterlage einen Hiatus: Das Mitteldevon des Breuschtals ruht wahrscheinlich diskordant auf steilgestelltem Steiger und Weiler Schiefer (BückING, 1. c., S. 5). Die Unterlage der unterkarbonischen Gesteine der südlichen Vogesen ist nicht mehr feststellbar, da Granitlakkolithen sich bis zu diesen durchgeschmolzen haben.

1) Auf der Versammlung der Geologischen Vereinigung in Gießen im März 1920 wandte Prof. WrLckens ein, daß nach einer ihm von Frau Dr. RicHTER, Frankfurt a. M., gemachten Mitteilung Dr. LEIDHowD (früher Assistent in Straßburg) im Buntsandstein der Vogesen ein Geröll mit einwandfrei unterdevonischen Fossilien bestimmt habe. Meines Erachtens ließe sich diese Tatsache auch mit einem Wandern des Gerölls aus rheinischen Gebieten nach S erklären. Autochthon kann ja das Material des Buntsandsteins in seiner Gesamtheit ohnehin nicht sein. Das genannte Gesteinsstück enthielt nach einer liebenswürdigen Mitteilung von Hermn Dr. Cu. Leinhold, Wietze, Spirifer hystericus Schloth., Choneten, und Rensselärien aus der Verwandtschait der strigiceps, was auf ein Aquivalent des Taunus. quarzites hindentet. - Ferner beschreibt A. Fvors (Jahrb. preuß. geol. Landesanst. Bd. 33. II. 1912. Nr. 63) eine von A. LEPPLA als Geröll im mittleren Buntsandstein der Grube Velsen bei Gr.-Rosseln (Bl. Dudweiler) gefundene Centronella taunica A. Fuchs in Taunusquarzitgestein. - Weiter liegt in der Straßburger Sammlung ein Buntsandsteingeröll mit Orthothetes umbraculum und ein Spirifer. speciosus, der schon von E. BENBows (Abh. zur geol. Spez.-Karte v. Els.-Lothr. Bd. I. S. 565) erwähnt wird. - Ich verdanke Hinweise auf diese Funde den Herren Dr. CL. Lwio. HOLD und Dr. RUD. RICFTER. - Ein autochthones Vorkommen von Unterdevon in den Vogesen wird auch dadurch nicht wahrscheinlich gemacht, was im übrigen für obige Überlegungen bedeutungslos ist. 
Aber die Komponenten schon der älteren Konglomerate gestatten einen Schlub auf die Zusammensetzung der damaligen Hochgebiete. Gneis, Gabbro, Serpentin müssen daran beteiligt gewesen sein (L. v. WERVEKk, Mitt. geol. Landesanst. Els.-Lothr. Bd. 3. 1892). Ähnlich liegen die Verhältnisse im südlichen Schwarzwald. Hier ist Granit an der Zusammensetzung der Kulmkonglomerate beteiligt (S. v. BuBNoFr, 1919).

Alle Vorkommen von Oberkarbon in Schwarzwald und Vogesen bieten das gleiche Bild. Keines zeigt eine lückenlose Sedimentation während des ganzen Unterkarbons, keines ruht auf unterkarbonischem Sediment, alle dagegen mit mehr oder weniger größerem Hiatus auf weit Älterem, meist Gneis und Granit. H. Eck erkannte, daß das Unterkarbon von Hohengeroldseck bei Lahr, nach Sterzer (Mitt. Bad. geol. Landesanst. Bd. 5. 855) vom Alter der Oberen Ottweiler Stufe, auf Gneis lagert (Geogn. Karte d. Umgeb. v. Lahr m. Erl., 1844). Die der unteren und mittleren Saarbrücker Stufe (STERzEL, l. c.) entsprechenden flözführenden Bildungen von Diersburg-Berghaupten bei Offenburg sind nach H. Ziervogess Untersuchungen zwischen Gneis und Granit eingepreßt (Mitt. Bad. geol. Landesanst. Bd. 8. 1914. 1), und das Baden-Badenen Oberkarbon, Ottweiler Alter nach Strenzer (l. c.), legt sich diskordant auf gneisartige Schiefer und auf Granit (H. THüracr, Z. d. D. g. G. Bd. 65. 1913. 489). Ähnliches läßt sich am Oberkarbon der Vogesen beobachten. Kleine Fetzen liegen bei, Thannenkirch im Glashüttental und in der Umgebung der Hohkönigsburg auf Granit; oberhalb Bonhomme und im oberen Lebertal liegen oberkarbonische Konglomerate zwischen Gneis und Granit eingepreßt (BenEcke, Geol. Führer d. d. Elsaß. 1900. 22). Auch die bekannten Kohlenvorkommen von Roderen und St. Pilt südwestlich Schlettstadt, nach STrerzer aus der Utbergangsperiode von Saarbrücker zu Ottweiler Zeit, ruhen auf Granit (L. v. Werveke, Philom. Ges. v. Els.-Lothr. Bd. 2. 1898-1902, 244); das Oberkarbon von le Hury am Ful des Rammelsteins auf Gneis (ebenda, 245). Die Laacher Schichten im Urbeiser Tal aus jüngster Ottweiler Zeit sind auf Granit gelagert (Büorrvg, Mitt. geol. Landesanst. Els.-Lothr. Bd. 12. 1918. 7).

Fast allen diesen Bildungen gemeinsam ist das Vorkommen von Arkosen, ein Beweis, daß feldspatreiche Gesteine das Abtragungsgebiet aufbauten. Das Gleiche wird erwiesen durch das Vorkommen von Kammgranit und anderen Granitarten in den Konglomeraten des vogesischen Oberkarbons (Benecke, Führer d. d. Elsaß. 1900. 58). Unterkarbon noch metamorphosierend, waren sie in Ottweiler Zeit bereits entblößt.

Uberblickt man diese Tatsachen in ihrer Gesamtheit, so bedeuten sie für den Bereich der Oberrheinischen Massive folgendes: Das Schicksal des.Gebietes in silurisch-devonischer Zeit ist nicht mit Sicherheit zu rekonstruieren. Dauernde Sedimentation ist sehr unwahrscheinlich. Unter dem Breuschtal-Mitteldevon fehlt das Unterdevon, 'das anstehend im ganzen Bereich nicht nachgewiesen 
wurde. Mitteldevonische Meeresbedeckung herrschte in Süd- und Mittelvogesen, in letzteren ausgesprothen landnahe; griff vielleicht in rechtsrheinische Gebiete über (Baden-Baden, Odenwald). Ein Nachweis ist dafür nicht zu erbringen. Für das Oberdevon liegen die Verhältnisse ähnlich wie für das Unterdevon. An der Basis des Unterkarbons Fehlen oberdevonischer Sedimente. Das scheint für oberdevonisches Hochgebiet $z \mathfrak{s}$ sprechen. Bereits früher habe ich darauf hingewiesen, $\mathrm{daB}$ die fazielle Ausbildung des Oberdevons in Mitteldeutschland für diese Zeit ein süddeutsch-böhmisches Festland fordert (A. BorN, N. Jahrb. f. Min. Beil.-Bd.34. 1912. 566). O. H. Schundewole ist neuerdings auf Grund seiner Studien in Fichtelgebirge und Ostthüringen zu gleicher Erkenntnis gelangt (Senkenbergiana. Bd. 2. 121). - Unterkarbon kam nur im Süden zur Ablagerung. Eine ehemals größere Ausbreitung über den ganzen. Bereich halte ich für ausgeschlossen; dann müßte mindestens das älteste Oberkarbon, das von Diersburg-Berghaupten mit seiner Waldenburgisch-Saarbrücker Mischflora auf Unterkarbongestein und nicht auf Gneis und Granit ruhen.

Für die Beurteilung. des übrigen Teiles von Süddeutschland stehen wenige natürliche Aufschlüsse und eine Reihe von Tiefbohrungen zur Verfügung. Die Schichtenlücke wird hier inmer größer. Im Süden hat auf schweizerischem Gebiet eine Tiefbohrung bei Zurzach unter oberem Buntsandstein sofort zersetzten Granit erschlossen (W. DeEore, Geologie v. Baden. Bd. 1. 92, Anmerk.). Am östlichen Schwarzwaldrand, bei Schramberg, traf eine Bohrung unter ältestem Unterrotliegendem ebenfalls Granit (Sa uer, Erl. zu Bl. Schramberg). Gleiches Ergebnis hatte eine Bohrung bei Ingelfingen im Hohenloheschen (E. FraAs, Begl. Worte zur geol. Karte v. Württbg. 1 : 50000 , Bl. Mergentheim, Künzelsau usw. 1892. 10). Im Norden, am Rande des Saar-Saale-Grabens das gleiche Bild, bei Kissingen Oberrotliegendes auf Granit (v. Ammon, Erl. Bl. Kusel geol. Karte v. Bayern. 1910. 64). Auch die Hegau-Auswürflinge bergen außer Sedimenten bis herab zur Trias nichts anderes als Kristallines (TH. BuRI, Ber. Naturf. Ges. Freibg. 18. 1911. 72). Die Tuffröhren der Schwäbischen Alb haben als ältestes Sediment Buntsandstein oder Rotliegendes, dessen Komponenten Gneis und Granit sind, gefördert (M. BRäUHÄUSER, Jahresber. Vaterl. Ver. f. Natk. in Württemberg. 74. 1918. 213). Das Nördlinger Ries birgt als ältestes den Keuper. Und im Osten taucht das kristalline Gestein der böhmischen Masse aus einem Deckgebirge auf, das teils aus Rotliegendem, teils aus verschiedenen Stufen des Mesozoikums besteht.

Diese Tatsachen verkünden ein kristallines Hochland, das zum großen Teil präpermisçb bestand, z. T. jedoch erst später von den mesozoischen Transgressionen überwunden wurde. Die Lücke Gneis- bzw. Granit-Rotliegendes läßt an sich wenig Möglichkeit für die Beurteilung der Frage zu, ob etwa paläozoische Bedeckung be- 
stand oder nicht. Die Möglichkeit einer anfänglichen Sedimentation mit nachfolgender Abtragung ist natürlich immer gegeben.

Ein Umstand bleibt jedoch zu bedenken. Alles, was Süddeutschland - abgesehen von den Oberrheinischen Massiven - an präpermischem Gestein geliefert hat, gehört nicht in die Rand-" und Dachzonen der Granitlakkolithe. Was die Bohrungen im Untergrunde Süddeutschlands erreicht haben, ist Granit. Etwas mannigfaltiger ist das Material, was vulkanische Tätigkeit im Hegau, im schwäbischen Maargebiet und im Ries gefördert hat. Paläozoisches Gestein fehlt überall. Im Hegau ist mittlerer Buntsandstein das älteste sicher beobachtete Sediment (Тн. BurI, Ber. natf. Ges. Freiburg. Bd. 18. 1911. 89). Das reichhaltige Grundgebirge enthält nach ERB (Vierteljahrsschr. natf. Ges. Zürich. 45. 1900. 55) Zweiglimmergranit, Biotit- und Biotithornblendegranit, Aplite, Glimmersyenite, Biotitgneise und Glimmerschiefer. Das sind Gesteine, die mit denen des Schwarzwaldes gewisse Übereinstimmung aufweisen. Doch fehlen alle Vertreter aus der Zone der exomorphen Kontaktmetamorphose.

Nicht weniger mannigfaltig ist die Serie der kristallinen Gesteine unter den Auswürflingen der Schwäbischen Alb-Vulkane (H. ScHWARZ, Jahresheft Vaterl. Ver. f. Natk. in Württbg. 61. 1905. 227). Zehn Gneisarten, von Graniten Pinit- und Mariolitgranit; an Ganggesteinen Aplit und Kersantit. An Sedimenten ist Rotliegendes, dann Mittlerer Buntsandstein das Älteste. BRÄUHÄUSERs interessante Studie wies das nach (Jahresber. Vaterl. Ver. f. Natk. in Württbg. 74. 1918. 212). Nach seinen Untersuchungen gibt die Tuffzusammensetzung nicht nur Aufschluß über die Art des ehemals unter den Tuffröhren befindlichen Gesteinkomplexes, sondern, wenigstens bezüglich der kristallinen Gesteine Aufschluß über einen weit größeren Raum. BRÄUHÄUSER erbrachte aus der Abrollung der kristallinen Komponenten und aus ihrer Verbreitung den Wahrscheinlichkeitsbeweis, daß eine Rotliegendsenke, NO-SW von Reutlingen nach Nürtingen verlaufend, von einer Reihe von Explosionen durchschlagen wurde. Dieser Senke entspricht ein weites Einzugsgebiet, das nunmehr in seiner Zusammensetzung der Beurteilung zugänglich ist. Bei den Gneisen betont Schwarz die Anklänge an den Bayrischen Wald. Mit dem Schwarzwald bestehen solche nur bezüglich der Granittypen, die überhaupt universellen Charakters. Die von ScHWarz u. a. bereits erkannte Utbereinstimmung mit dem Grundgebirge des Nördlinger Ries und seiner Umgebung haben R. LöFFIERS Studien bestätigt (Jahresh. Ver. f. vaterl. Natk. Württbg. . 68. 1912. 109). Das betrifft besonders die Gneise. Da die Feststellung kontakt-metamorph veränderter Sedimente von Bedeutung ist, darf das Auftreten eines Gesteins nicht unerwähnt bleiben, das sich auf der Alb an einigen Punkten des Ries gefunden hat. Es handelt sich um ein quarz- und feldspatfreies Gestein, an dessen Aufbau Pinit als Grundmasse, daneben Granat, Biotit, Magnetit und Silimanit beteiligt ist, 
ein Gestein, das Lörfler (1. c.) als Schiefer bezeichnet. Das Gestein würde eventuell einer sehr kontaktnahen Zone angehören.

Soweit wir durch die natürlichen und künstlichen Tiefenaufschlüsse uns ein Urteil über den kristallinen Untergrund von Süddeutschland zu bilden vermögen, ergibt sich folgendes: Was von kristallinem Gestein bekannt ist, stimmt mehr mit Böhnuisch-Bayrischem Wald, als nit Schwarzwald überein, besonders bezüglich der Gneise, obwohl auch hier kein Gegensatz zum Schwarzwald besteht. Mindestens schon in Rotliegender Zeit bestand hier ein Gneis-Granit-Hochgebiet. Nimmt man für paläozoische Zeit Sedimentation an, so mub schon frühzeitig, mindestens in unterkarboner Zeit, die Abtragung eingesetzt haben, da sonst nicht ältestes Rotliegendes auf kristallinem Gestein aufliegen könnte. Das Fehlen von Spuren aus der Zone der exomorphen thermischen Kontaktmetamorphuse könnte vielleicht dahin gedeutet werden, daß das Gebiet von Mittel-und Ost-Süddeutschland schon eher einer Abtragung unterlegen ist, als das der Oberrheinischen Massive. Das Dach der Lakkolithen war dort völlig beşeitigt, als das Rotliegende zur Ablagerung kam, im Gegensatz zu Odenwald - SchwarzwaldVogesen, wo Gesteine der Dachzone bei Beginn der Rotliegendzeit noch mehr oder weniger erhalten sind. Man darf daraus vielleicht auf ein um so früheres Aufsteigen des heute versunken liegenden Untergrundes von Süddeutschland schließen.

Kombiniert man die so von versuhiedenen Gesiuhtspunkten aus gewonnenen Resultate, erhält man etwa folgendes Bild vom Zustand Süddeutschlands zur Zeit des Beginns varistischer Gebirgsbildung:

Wiederholt im Verlauf des Paläozoikums nicht im Bereich der Sedimentation, sondern der Denudation, so im Mitteldevon: Sand in Böhmen, landnahe Breccien und Kunglomerate nebst Saumriffen im Breuschtal. Hier, wie W. Deecke bereits betonte, nur lokales Transgredieren des Mitteldevons von W und SW (Geul. v. Baden. Bd. 1. 154). Gneis und Granit als Komponenten der grobterrigenen Bildun zen des Unterkarbons der südwestdeutschen lokalen Geosynklinale (Südschwarzwald und -vogesen). Die beständige Auflagerung von Oberkarbon auf Granit und Gneis in Suhwarzwald und Vogesen als Beleg, daß der Sedimentmantel, auch der kontaktmetamorpher Umwandlung, bereits zum großen Teil beseitigt ist. Als während unterkarbonischer Zeit (im südlichen Schwarzwald) orogenetische Bewegungen einsetzten and um die Wende von Unter - und Oberkarbon umfassender auf traten, lag der größte Teil von Süddeutschland, südwestlichste und einige westliche Teile viellejcht ausgenommen, als zumeist kristallines Hochgebiet mit wenigen Resten sedimentärer Bildungen im Bereich der Abtragung; SW-NO streichende terrestre Senken mit lokaler Bedeutung sammelten den 
Hochlandschutt; im großen ganzen wurde er nach NW in die SaarSaale-Senke und in zentralböhmische Gebiete, die sich als Geosynklinale betätigten, abgeschüttelt. - -

Die Orogenese, die in karbonischer Zeit mit Druck von SO nach NW einsetzte, traf in Süddeutschland einen Gesteinskomplex an, der in seiner Zusammensetzung stark abwich von dem der Rheinischen Masse. Im Bereich der letzteren die mächtige Schichtenfolge einer Geosynklinale in mindestens obersilurischer devonischer und unterkarbonischer Zeit, eine marine Schichtenfolge von mindestens $2-3000 \mathrm{~m}$ Mächtigkeit, diese nur lokal durch episodische Hochgebiete herabgemindert, also ein Gebiet, in dem die kristalline Unterlage der Geosynklinale um den Betrag der abgelagerten Schichtenmächtigkeit in die Tiefe versenkt wurde. Physikalisch und schematisch gedacht ein System zahlreicher dünner parallel gelagerter Platten verschiedener Riegheit. im Bereich Süddeutschlandis dagegen ein kristalliner Komplex, vorwiegend aus Gneis und Granit bestehend, mit Resten kontaktmetamorpher, z. T. eingeschmolzener und injizierter Lakkolithdächer. Nur im Süden lag eine kleine Geosynklinale diesem kristallinen Komplex angegliedert.

Es ist selbstverständlich, daß diese beiden Gesteinskomplexe gegenüber orogenetischem Druck sich verschieden verhalten mußten, und daß die Anpassung an die neuen physikalischen Bedingungen als Gleichgewichtszustand Formen erzeugen mußte, die wesentlich voneinander abwichen.

Die Gefügigkeit gegenüber orogenetischem Druck hat H. STILLE als Mobilität, ihr Gegenteil als Stabilität bezeichnet (Geol. Rundschau. Bd. 8. 1917. 101). Vom Gesichtspunkt der Faltbarkeit ist' es selbstverständlich, daß sich das kristalline Hochgebiet des Südens gegenüber orogenetischem Druck relativ stabil verhalten mußte. Vor allem darf man hier kein echtes Faltengebirge erwarten, wie im Bereich der Rheinischen Masse; dazu fehlten von vornherein die entsprechenden Materialien. W. DEECKE hat eingehend auf diese Tatsache hingewiesen (Geol. v. Baden. Bd. 1. 151).

Aus der Unterlage und den Gesteinskomponenten von Unterkarbon, Oberkarbon und. Unterrotliegendem läßt sich in Süddeutschland eine vorwiegend aus Gneis und Granit sich aufbanende Landoberfläche für die Zeit der karbonischen Phasen der varistischen Orogenese rekonstruieren. Es muß endgültig mit der Vorstellung gebrochen werden, es könnte ein Teil der wieder entblößten Schwarzwald- und Vogesengneise der varistischen Dynamometamorphose ihre Entstehung verdanken. Diese Gesteinskomplexe lagen zu jener Zeit an der Oberfläche, unterlagen nicht den Gesetzen der Tiefe, der Gesteinsumwandlung durch Ummineralisierung und Neuorientierung der Komponenten, gehörten der Zone of Katamorphism, nicht der of Anamorphism an. Sie waren als Gneise fertig und konnten nur mechanisch umgewandelt werden. Es waren 
nur rein kataklastische Vorgänge möglich, da die Ausweichsmöglichkeit nach oben stets gegeben war. Auch die Faltung der Gneise muß älter sein, da die physikalischen Bedingungen für bruchlose Faltung so oberflächennahe nicht gegeben sein konnten! Die Anordnung der Falten der Gneise in varistischer Richtung darf nicht in diesem Sinne gedeutet werden. Es ist umgekehrt vielmehr anzunehmen, daß die varistische Faltung, soweit eine solche in den Oberrheinischen Massiven überhaupt vorhanden, in bezug auf die der SW-NO streichenden Gneise posthum. Die Frage der zeitlichen Bildung der Gneise bedarf noch der Klärung. W. Dewcke ist diesem Problem nachgegangen (Geol. v. Baden. Bd. 1. 57-59). Hier steht die Frage nicht zur Diskussion. Wir stellen lediglich fest, daB sie einer varistischen Orogenese nicht. angehören.

Die varistische Orogenese traf also einen Komplex an, der sich zusammensetzte vorwiegend aus vergneisten alten Eruptiven und Sedimenten, die durch Granitintrusionen eine weitere Versteifung erfahren hatten. Sedimentäre, z.T. eingeschmolzene Reste waren sporadisch vertreten. $\mathrm{Ob}$ das Ganze ohne jede texturelle Gliederung war, ist schwer zu sagen. Möglich wäre eine prävaristische Orogenese, die sich vielleicht in den Weiler und Steiger Schiefern zu erkennen gäbe. Das Ergebnis orogenetischen Drucks auf eineh derart struierten starren Körper ist die Bildung kataklastischer Formen in jeder Dimension. Entstehung nur mikroskopisch erkennbarer kataklastischer Gesteinskomplexe wie z. B. des Granits von Altglashütten (S. v. BuBnoFr, Mitt. Bad. geol. Landesanst. 7. 1912) einer seits, die Bildung großer Störungs - und Quetschzonen, an denen sich Horizontalverschiebungen und Schuppungen vollzogen, andrerseits. Was an Sediment vorhanden war, wurde gefaltet, besonders im Süden von Schwarzwald und Vogesen, wo die Bildungen der südwestlichen Unterkarbongeosynklinale zur Verfügung standen. Aber bemerkenswerter Weise kam es auch hier nicht zu intensiven Faltungsvorgängen, wie im Bereich der Rheinischen Masse. Isoklinale, etwa nach $\mathbf{N}$ überkippte Faltenelemente fehlen. Wir treffen normale oder nur schwach schiefe Mulden an, mit mittleren Einfallswinkeln und von größerer Spannweite. Dabei ist bei manchen der Zweifel exlaubt, ob es sich nicht um Bildungen einer jüngeren, vielleicht tertiären Orogenese handelt. Einen echten Sattel bildet das Mitteldevon (?) der südlichen Vogesen zwischen Chagey und Chennebier (nördlich Héricourt), ein normaler Sattel, der nach meiner Beobachtung mit einem Einfalls-

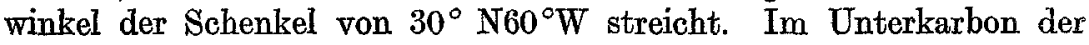
Südvogesen läßt sich von echter Faltung, wohl infolge der drängenden Kräfte der aufsteigenden Granitlakkolithen nichts erkennen. Im südlichen Schwarzwald wies S. v. BuBNoFF eine intraunterkarbone Bodenbewegung nach, der eine spätere postunterkarbone folgte, deren echte, wenn auch weitgespannte Faltengebilde im Lènzkircher Graben erhalten 
sind (Jahresber. Oberrhein. geol. Ver., N. F. 8. 1919. 28 und Eclogae, Bd. 14. 1916. 246).

Der Betrag des Zusammenschubs ist überall nur gering; die Position des kristallinen Untergrundes, $d . h$. seine oberflächennahe Lage unter einer mehr oder weniger zusammenhängenden geringmächtigen Sedimentdecke blieb auf die Faltung letzterer nicht ohne Einfluß. Der faltungsfeindliche starre Untergrund wirkte schützend auf seine relativ dünne Sedimentdecke. Das oberkarbone Deckgebirge aber wurde durch den kristallinen Untergrund, der selbst nur durch Bewegung größerer Klötze oder Massen gegeneinander auf faltenden Druck reagieren konnte, zu muldenartigen Einquetschungen an Zonen, wo zwei kristalline Blöcke gegeneinander bewegt wurden, veranlaßt. Vielleicht ist die Oberkarbonmulde von Diersburg-Berghaupten, die erzgebirgisch streichend zwischen Granit und Gneis eingepreßt ruht (ZIEPvoGEL, Mitt. Bad. geol. Landesanst. Bd. 8. 1914. 1) in dieser Weise zu verstehen. Auch für die gestörte Lagerung des Oberkarbons von Bonhomme und im oberen Lebertal (Bückrng, Führer d. d. Elsaß. 1900. S. 22) wäre hiermit eine Erklärung gegeben ${ }^{1}$ ).

Innerhalb des kristallinen Körpers äußerten sich die Spannungen in der Weise, daß streichende und Querstörungszonen entstanden, an denen Verschiebungen, teilweise Überschiebungen sich vollzogen. In neuester Zeit haben S. v. BuBNoFrs Untersuchungen im südlichen Schwarzwald über derartige Vorgänge Klarheit gebracht (Eclogae, Bd. 14. 1916. 246). 'Die große zentralschwarzwälder Gneisscholle wirkte bei der varistischen Auffaltung als stabile Masse hemmend und ablenkend auf die im Süden aus der Unterkarbongeosynklinale' aufsteigenden Faltenzüge. Diese Masse, ein altes vorkarbonisch versteiftes Hochgebiet, wurde in toto bewegt und schob sich nach $S$ an und auf das Unterkarbon hinauf, BuBNoFrs südschwarzwälder Utberschiebung. Das ist das einzige gut bekannte Beispiel vom Verhalten des süddeutschen kristallinen Hochgebietes zur Zeit der varistischen Orogenese.

In allergrößtem Gegensatz zum Ergebnis dieser Orogenese im süddeutschen Hochgebiet steht das Ergebnis im Bereich der varisti schen Geosynklinale, wie es besonders aus dem Gebiet der Rheinischen Masse bekannt ist. Was hier vorliegt, ist der Typ eines Faltengebirges hochentwickelten Stadiums. Der Grad der

1) Ganz analoge Verhältnisse hat W. BRöGGER (Sil. Etage 2 und 3 im Kristianiagebiet. 1882. S. 216) im Silurgebiet von Kristiania beobachtet. Hier ruht geringmächtiges Silur auf kristallinem Grundgebirge. Ersteres hat auf den Faltungsdruck der kaledonischen Orogenese mit einer disharmonischen Faltung in Abhängigkeit von der Mobilität des Gesteins reagiert. Ganz abweichend verhielt sich das Grundgebirge. Von diesem wurden nur größere Partien, innerhalb welcher sich keinerlei Faltungs- oder Stauchungserscheinungen nachweisen ließen, aufgepreßt. Die Bewegung vollzog sich in der Weise, daß die großen Gesteinskomplexe durch wachsende Drehung um eine der Streichrichtung der Silurfalten parallele Drehungsa.chse gehoben wurden. Die Verhältnisse liegen nicht unähnlich im Sohwarzwald. 
Faltbarkeit, der dem gesamten Gesteinskomplex a priori zukam, war relativ hoch. Eine ursprünglich vielleicht vorhanden gewesene kristalline Unterlage der Geosynklinale, welche faltungshemmend hätte wirken können, war durch epirogenetische Bewegungen in große Tiefe gewichen und durch mobileres, sedimentäres Material ersetzt worden, das Gebiet also im Sinne von $H$. STrLL刃 tiefgründig geworden. Man könnte annehmen, daß eine Serie paralleler Platten verschiedener Zusammensetzung und Riegheit, wie sie schematisch gedacht durch die Sedimente der Rheinischen Geosynklinale dargestellt werden, einen relativ stabilen Charakter besäße. Dieser Vorstellung entsprechen jedoch die tatsächlichen Verhältnisse nicht ganz. Zunächst liegen die Sedimente a priori nicht horizontal, sondern flach geneigt gegen das Muldeninnere, mit um so größerem Fallwinkel, je kleiner bei gleicher Tiefe die Spannweite des Sedimentationsraumes ist; und diese ist um so geringer, je häufiger alte Kerne als Hochgebiete die große Geosynklinale in Teilbecken gliedern. Als solche betätigten sich, wenigstens zeitweilig, in der Rheinischen Geosynklinale u. a. der Siegerländer Block (WEDEKIND), die Hörre und der Taunus (J.AnLBuRg). Auf diese Weisé entstand primär eine sanfte Wellung der synchronen Sedimenttafeln, die durch epirogenetisches Sinken der Sedimentationsräume bzw. durch Aufsteigen der Hochgebiete noch gesteigert wurde. Damit fiel von vornherein ein die Faltung hemmendes Moment aus: die anfängliche Starrheit einer ebenen Schichtentafel, welche von Faltungsdruck in ihrer Ebene betroffen wird. SMoLUoHowsKI kam bei seinen Untersuchungen auf mathematisch-mechanischer Grundlage über die Entstehung von Faltengebirgen (Anzeiger d. Akad. d. Wiss. Krakau, math.-phys Kl. 1909. II. S. 3) zum Ergebnis, daß der Druck zur Fortsetzung einer Faltung geringer als der zur Finleitung eines Faltungsvorganges an noch ungefalteten Schichtentafeln sei. Diese anfängliche Starrheit dürfte kaum vorgelegen haben, um so weniger, als ja ältere Verbiegungen aus oberdevonischer und unterkarboner Zeit vorgearbeitet hatten. Man darf also das Gebiet der varistischen Geosynklinale, speziell im Bereich der Rheinischen Masse, als einen gegen Faltungsdruck relativ mobilen Körper ansehen.

Orogenetisch stellt der Komplex der Rheinischen Masse, der am Ende des Paläozoikums vorlag, keine Einheit dar. Ohne das Problem der Auffaltung des varistischen Bogens, seine Zusammensetzung aus verschiedenzeitlichen Komponenten, den Einfluß alter Hochgebiete am Rande und Kerne im Inneren, die Zusammenhänge der vulkanischen Vorgänge mit den Faltungsphasen hier anzuschneiden, möchte ich auf gewisse Eigentümlichkeiten im Verhalten tektonischer Einheiten des rheinischen Sektors zur Zeit der varistischen Orogenese hinweisen. Die mechanischen Unterlagen bieten die obigen Ausführungen über süddeutsches Hochgebiet und rheinische Geosynklinale und ihr verschiedenes orogenetisches Verhalten. 
Die Anzeichen präkarbonischer orogenetischer und epirogenetischer Bodenbewegungen aus dem Bereich der Rheinischen Masse mehren sich mit jeder zusammenfassenden Arbeit über Sedimentation und Tektonik im Bereich des Rheinischen Schiefergebirges. Ich gedenke nur der Studien A. Dencruanns im Siegerland über Unterdevonische Bodenbewegungen orogenetischer Natur, R. WEDEKINDs Feststellung eines Siegerländer Blockes für die Zeit des Oberdevon, R. ScHINDEwoLFs gleichsinniger Beobachtungen für das Mitteldevon; der zusammenfassenden Betrachtungen R. WEDERINDs über Transgressionen im Oberdevon und Unterkarbon (Jahresber. Niedersächs. geol. Ver. 1914, S. 34); J. AhrbuRgs zusammenfassender Arbeiten im Dill- und Lahngebiet, die mittel- and oberdevonische und unterkarbone Bodenbewegungen zum Ergebnis hatten. Soweit es sich um echte orogenetische Vorgänge handelt - die Entscheidung darüber ist nicht immer einwandfrei möglich -, kann man diese Zwischenspiele einem zu echter Gebirgsbildung führenden Vorgang als Vorläufer oder Nachzügler angliedern. Die Entscheidung darüber wird davon abhängen, ob in diesen Vorgängen sekundärer Art die Streichrichtung einer vorhergehenden oder einer folgenden Orogenese zum Ausdruck kommt. Innerhalb der Rheinischen Masse würde man festzustellen haben, welche der unterdevonischen bis unterkarbonischen orogenetischen Bewegungen etwa einer kaledonischen und welche einer varistischen Orogenese angehören. Die Lösung, der an anderer Stelle nähergetreten wird, wird dadurch erschwert, daß auch die Streichrichtung der kaledonischen Orogenese sich um eine mittlere Linie NO-SW bewegt.

Sieht man von allen Vorphasen varistischer Gebirgsbildung bis in das Unterkarbon ab, so gelten zwei Phasen als wesentlich für die Auffaltung des Varistischen Bogens, wenigstens im Bereich des Rheinischen Sektors: eine erste Hauptphase um die Wende von Unter- und Oberkarbon, eine zweite Hauptphase nach Abschluß des Oberkarbons. Die Rheinische Masse gilt als Typ eines Beispiels für das Wandern der Gebirgsbildung (H. STILLE): Die erste Hauptphase soll sich in südlichen Teilen betätigt haben, die zweite Phase soll die nördlichen äußeren Teile den sïdlichen angeschlossen haben. Die strenge Beweisführung würde den, Nachweis folgender Lagerungsverhältnisse erfordern: Im S Oberkarbon diskordant iiber gefaltetem Unterkarbon und Ailterem; im N Unter- bzw. Oberrotliegendes diskordant über Oberkarbon. Weder das eine noch das andere Profil ist jedoch als Nachweis beizubringen.

Diese Tatsache kann nicht iiberraschen. H. StiLle hat darauf hingewiesen, daß alle orogenetische Bewegung in bezug auf das Niveau des Meeres eine Hochbewegung ist. Sie ist eire Ausweichbewegung nach oben. Es muß also jedes von orogenetischer Bewegung ergriffene Gebiet nach oben bewegt, dem Bereich bisheriger Sedimentation entzogen, in ein totes Stadium oder in den Bereich der Denudation emporgehoben werden. Das ist die selbstverständliche Folge stark ausgeprägter 
Orogenese. Die mit der Hebung verbundene Abtragung verhindert, daß später wieder einsetzende Sedimentation sich lückenlos anschließt. Im übrigen darf man nicht erwarten, postorogenetische Sedimente dem gefalteten Komplex auf-, sondern höchstens angelagert zu finden. Denn die Sedimentation dauert in den nicht orogenetisch bewegten Gebieten an bzw. wird dort neu belebt. Es ist eine nicht mehr als zufällige Erscheinung, daß im Bereich der ganzen Rheinischen Massen nirgends Oberkarbon den präoberkarbonisch gefalteten Gebieten angelagert ist, dab also die zeitliche Festlegung der mittelkarbonen Orogenese nicht mit Sicherheit möglich ist. Denn alles Oberkarbon von Westfalen (vgl. die preuß. geol. Spez.-Karten Iserlohn 1911, Hohenlimburg 1911, Hagen 1911, Menden 1911), Rheinland, Belgien und Nordfrankreich ruht konkordant auf Unterkarbon, das von der ersten Phase nicht ergriffen wurde, kommt also zur Altersfeststellung dieser nicht in Frage. Das Oberkarbon des Saargebietes tritt über Tage mit dem Südrand der Rheinischen Masse nirgends in Kontakt, da es überall im $N$ von transgredierendem Rotliegenden überlagert wird. Da aber Unterrotliegendes im wesentlichen konkordant auf Oberkarbon ruht, ist somit indirekt auch die grobe Diskordanz zwischen Oberkarbon und gefaltetem Rheinischen Schiefergebirge erwiesen. Zur genaueren Altersfestlegung ist noch ein zweiter indirekter Schluß notwendig. Linksrheinisch tritt im Hunsrückgebiet kein Unterkarbon mehr auf. Diese Regionen waren damals bereits Hochgebiet, eventuell schon frïher. Was hier von Oberkarbon subterran überlagert wird, ist höchstens Mittel- und Unterdevon. Aus dem Umstand, daß rechtsrheinisch Oberdevon und Unterkarbon in annähernd gleicher Weise wie Älteres der Faltung unterlagen, sind wir berechtigt zu schließen, daß die Orogenese an der Wende von Unter- und Oberkarbon einsetzte. Immerhin bleibt dabei der subjektiven Entscheidung dés Einzelnen mancherlei überlassen und es ist von Vorteil, sich bewußt zu bleiben, auf wie unsicherer Basis Theorien ruhen, die zum sicheren Bestand unserer Lehrbücher gehören.

Aus dem Umstand, daß am Nordrand der Rheinischen Masse Oberkarbon konkordant auf Unterkarbon und dieses konkordant auf Oberdevon ruht, geht hervor, daß die mittelkarbonische Orogenese diese nördlichen Gebiete nicht erreicht hat. Die Grenze des Wirkungsbereiches dieser ersten varistischen Phase liegt südlicher, im gefalteten Komplex der Rheinischen Masse. Zur Feststellung dieser Grenze dienen folgende Überlegungen: Erstens wäre zu untersuchen, ob die Streichrichtung der Falten im Gebiet erster und zweiter Phase völlig in Ubereinstimmung mit der nur zweiter Phase. Zweitens müßte in beiden Gebieten eine verschiedene Faltungsintensität erkennbar sein.

Bezüglich der Streichrichtung der Falten bestehen im Rheinischen Schiefergebirge Gegensätze, die rein lokal aus der Präexistenz älterer Kerne zu verstehen sind; daneben aber auch solche, die auf verschiedenartige Entstehung hindeuten. Den Versuch einer tektonischen Analyse 
des Rheinischen Schiefergebirges hat vor kurzem H. VogkL ${ }^{1}$ ) unternommen. Die differentielle Unterscheidung zwischen den beiden wichtigen Streichrichtungen, der älteren etwa $\mathrm{N} 35-40^{\circ} \mathrm{O}$ und der jüngeren N $60-70^{\circ} 0$, bedeutet einen Fortschritt. Aber ihre zeitliche Festlegung und die Unterscheidung einer "mittelkarbonen " und postoberkorbonen orogenetischen Phase bedürfen noch einer konsequenteren Durchführung. Als nördliche Grenze der Auswirkung der ersten, mittelkarbonen Phase kommt das Siegener Hochgebiet in Betracht, das Vogel als Siegen-Stadtberger Sattel bezeichnet. Zweifellos ist, daß die mittelkarbone Orogenese im wesentlichen eine südliche Hälfte der Rheinischen Masse erfaßte und eine nördliche unberührt ließ, obwohl vom Standpunkt der Faltbarkeit die nördlichen Gebiete die bevorzugten hätten sein müssen, wenn man die Lückenlosigkeit und Mächtigkeit des abgelagerten Materials als ausschlaggebendes Moment betrachtet. Vom Gesichtspunkt einer Auffaltung durch Gewölbespan. nung als Ergebnis von Schrumpfungsvorgängen ist diese tektonische Unberührtheit des nördlichen Geosynklinalgebietes unverständlich.

Die zweite, postoberkarbone varistische Orogenese ist zeitlich nicht einwandfrei festzulegen. Sie hat den Außenrand des Bogens nicht überall in gleicher Stärke betroffen. In Oberschlesien ist der Westflügel der Oberkarbonsenke relativ schwach gewellt, der Ostflügel fast unberührt geblieben. Am Nordrand des Rechtsrheinischen Schiefergebirges treffen wir einen höheren Grad von Faltungsintensität an, etwa vom Stadium des Faltenjura: meist normale Syn- und Antiklinalen, Uberkippungen sind selten, isoklinaler Bau fehlt noch. Weiterer Steigerung der Faltungsintensität begegnen wir im linksrheinischen Oberkarbon: steilgestellten Südflügeln der Mulden im Aachener Gebiet, überkippten Faltenelementen, Schuppungen und Überschiebungen, die nicht in den Beginn des Faltungsvorganges gehören (vgl. Bl. Aachen u. a.). Die Steigerung ins Extrem trifft man in der belgisch-nordfranzösischen Oberkarbonzone: vorwiegend isoklinaler Bau der Faltenelemente verbunden mit Überkippung nach $\mathrm{N}$, dazu Auswirkung in großen Überschiebungen mit weitem Deckentransport, wie bei der grande faille $\mathrm{du}$ midi. Im ganzen eine Steigerung der Faltungsintensität von SO nach W.

Daß diese Orogenese in das äußere Vorland auch Äquivalente des Rotliegenden abgeschüttet hat, darf man erwarten. Aber davon ist der Beobachtung heute nichts zugänglich. In dem zurzeit erschlossenen Gebiete transgrediert Zechstein diskordant auf gefaltetes Oberkarbon, wie die subterranen Aufschlüsse am Nordrand der Rheinischen Masse erwiesen, haben.

1) H. VoasL, Betrachtungen über den Aufbau des Rheinischen Schiefergebirges usw. Verh. naturhist. Ver. f. Rheinl. u. Westfalen. 76. Jahrg. 1919. S. 31. 
Zur Altersfeststellung der Faltung des Oberkarbons kommt dieses Außengebiet nicht in Frage. Die Faltung läßt sich lediglich als präzechsteinisch festlegen. Untersuchen wir die großen intermontanen. Rotliegendsenken des Varistischen Bogens: die Saar-Saale-KatzbachSenke, die Mittelsudetische Senke und das Böhmische Becken auf Anzeichen einer Orogenese in der Zeit zwischen Oberkarbon und Zechstein. Diese Frage habe ich kürzlich an anderer Stelle (Abh. Senckenberg. Naturf. Ges. 1921) eingehend erwogen, weswegen darauf verwiesen werden kann. Zunächst ist festzustellen, daß Unterrotliegendes stets konkordant Oberkarbon uiberlagert. Anzeichen orogenetischer Art intrarotliegenden Alters trifft man einmal im Erigebirgisehen Becken (Тн. BRANDEs, N. Jahrb. f. Min. B. B. 43. 1919. S. 223) in Form von NW streichenden Querbrüchen größerer Sprunghöhe postlebacher Zeit. Wesentlicher sind die orogenetischen Anzeichen im Saarbecken. A. LEPPIA berichtet (Festschr. 9. allgem. Bergmannstag 1904. S. 50-57) über Bruchtektonik aus Söterner Zeit. Neben diesen Vertikalbewegungen finden sich Auslösungen von horizontalen Spannungen: die Aufsattelung des Pfälzer oder Saarbrücker Hauptsattels, die parallel der Erstreckung des Saarbeckens NO-SW verläuft. Er ist nicht die Schöpfung einer orogenetischen Episode, sondern das Ergebnis säkular wirkender Spannungen (P. Kessler, Geol.-Pal. Abh., N. F. Bd. 13. 1914-15. S. 151-160). Auch in Söterner Zeit, in die ja die übrigen Anzeichen orogenetischer Bewegungen fallen, erfolgte eine Steigerung der Aufsattelung, ohne daB diesen Bewegungen eine besondere über das bisherige $\mathrm{Ma} B$ hinausgehende Intensität zuzukommen scheint. Im Anschluß hieran erfolgten einige zu Faltungserscheinungen führende Abgleitbewegungen an den Flügeln des Sattels (O. M. ReIs, Erl. BI. Zweibrücken bayr. geol. Karte 1913. S. 170 u. a. 0.).

Alles, was sonst an intrarotliegenden Dislokationen auftritt, sind schwache, sehr großwellige Schichtenverbiegungen des unteren und mittleren Rotliegenden, die von oberem Rotliegenden diskordant überlagert werden und die als Ergebnis epirogenetischer Bewegungen anzusehen sind.

Somit findet sich im ganzen Gebiet der intermontanen Rotliegendsenken kein quantitatives Äquivalent der ausgeprägten Orogenese am Außensaum des rheinischen Sektors. Und es zeigt sich wiederum das eigentümliche Bild, daß eine Zone erhöhter Faltbarkeit, wie die Saarsenke, die durch epirogenetische Bewegungen (Versenkung) von 6000 bis $7000 \mathrm{~m}$ Sediment ihre Faltbarkeit gesteigert hatte, von der orogenetischen, im N zur Faltung führenden Bewegung so gut wie unberührt blieb, wenn man überhaupt in den geringen intrarotliegenden orogenetischen Anzeichen ein zeitliches und genetisches Äquivalent der nördlichen Faltungsorogenese anerkennen will.

Ein Gebiet, das sich bei der "mittelkarbonischen " Faltungsphase als durchaus faltungsfreundlich erwiesen hatte, bleibt bei der nächsten 
Faltungsphase trotz inzwischen erhöhter Eignung für Faltungsvorgänge von diesen unberührt: eine tektonische Regionalauslese, die mit dem Prinzip der Gewölbespannung und Faltung der faltungsgeeignetsten Zonen des Gewölbes wiederum nicht in Einklang zu bringen ist.

Wir erkennen, die Auslese erfolgt nicht nach dem Prinzip der Faltbarkeit, sondern nach anderen Gesichtspunkten. Das deutet darauf hin, daß die faltende Kraft nicht jeweils das gesamte Gebiet des rheinischen Sektors ergriff, sondern nur die Teile betraf, in denen eine Faltungsorogenese zum Ausdruck kommt. Verschiedenaltrige Bewegungen des Untergrundes scheinen wellenartig Zonen der Erdhaut betroffen und gleichsinnig in Anspruch genommen zu haben.

\section{Der Wasserhaushalt des Inlandeises.}

\section{Von Alfred Jentzsch (Gießen).}

Zu verschiedenen Zeiten dẹr Erdgeschichte hat Inlandeis weite Landstrecken bedeckt. Ein Nachdenken über die physikalischen Möglichkeiten führt zu der Uberzeugung, daß auch in der kältesten Eiszeit neben dem Eise das Wasser nicht gefehlt, sondern in erheblichem Maße und in eigenartiger Weise an den geologischen Vorgängen der vereisten Gebiete mitgewirkt hat.

Im Zentrum der Vereisung mußte zur. Winterszeit die Lufttemperatur tief unter den Gefrierpunkt sinken; aber wenn überhaupt das Eis Geschiebe verfrachten sollte, so mußten zwischen dessen Zentrum und dem Außenrande des Eises Gebiete liegen, in denen mindestens zeitweise die Lufttemperatur höher als der Schmelzpunkt wurde.

Sonnenstrahlung hat nie ganz gefehlt. Selbst wenn über länderweite Gebiete Wolken und Nebel die Sonne verhüllten oder die Atmosphäre infolge beigemengter Dämpfe und Gase große Anteile der Wärmestrahlen verschluckte oder Ausstrahlung hohe Kältegrade herbeiführte, muß es auf der über mehrere Breitengrade sich erstreckenden Oberfläche eines Inlandeises mindestens zeitweise Stellen gegeben haben, in denen - wenn auch nur für Stunden oder Minuten - die Sonnenstrahlen genügten, um die Oberfläche des Eises zu schmelzen. Der Vorgang des Schmelzens mußte verstärkt und beschleunigt werden, wenn und wo immer Regen oder ein örtlich wärmender Luftstrom das Eis traf.

Ebenso mußte die nirgends fehlende Wärme-Ausstrahlung des Erdkörpers erwärmend auf die Sohle des Eises einwirken und dessen unterste Schicht schmelzen, so daß letztere mindestens zeitweise durch eine dünne Wasserschicht von dem festen Untergrunde getrennt blieb. Erwärmend und demnach schmelzend wirkte am Untergrunde auch die äußere und innere Reibung des Eises und die bekannte Diathermansie 